betreffenden auf der Faser abgelagerten Metalloxyd zum Farblack gebunden worden sind. Ein mit 1 bis 2 Proz. Farbstoff angesetztes Bad wird auf diese Weise vollständig ausgezogen.

Besonders kräftig färbt das 1.4. 5.-Trioxyanthrachinon in mehr oder weniger tiefen violetten Tönen $\mathrm{Ce}, \mathrm{Th}, \mathrm{Zr}, \mathrm{Y}, \mathrm{Gi}, \mathrm{Al}, \mathrm{Ur}, \mathrm{Cr}, \mathrm{Cu}, \mathrm{Bi}$, $\mathrm{TI}$, Fe-Beize, weniger intensiv $\mathrm{Co}, \mathrm{Ni}, \mathrm{Zn}, \mathrm{Cd}$, $\mathrm{Mn}, \mathrm{Pb}, \mathrm{Sn}$-Beize, gar nicht in Sb-Beize.
1. 4. 5. 8.-Tetraoxyanthrachinon färbt in blawen bis grünblauen Tönen kräftig $\mathrm{Th}, \mathrm{Zr}, \mathrm{Y}, \mathrm{Gl}, \mathrm{Cr}$, $\mathrm{Bi}, \mathrm{Fe}-\mathrm{Beize}$, etwas weniger intensiv $\mathrm{Ce}, \mathrm{Al}$, Ur, $\mathrm{Cd}$-Beize, fast gar nicht $\mathrm{Co}, \mathrm{Ni}, \mathrm{Cu}, \mathrm{Zn}, \mathrm{Mn}$, $\mathrm{Sb}, \mathrm{Pb}, \mathrm{Sn}, \mathrm{Ti}-\mathrm{Beize}$.

Die Folgerungen, welche v. Georgievics aus seinen Versuchen bezüglich des Einflusses der Hydroxylstellungen 1,5 und 1,8 in Oxyanthrachinonen auf deren beizenfärbende Eigenschaften zieht, treffen jedenfalls nicht zut.

\title{
Ueber die Oxydation von Kautschuk und Kautschukwaren.
}

\author{
Von Dr. Rudolf Ditmar, Graz. ${ }^{\text {) }}$
}

Eine der wichtigsten Frayen, welche den Kautschukfabrikanten beschäftigt, ist die Oxydationsfähigkeit des Kautschuks. Wenn man sagt, ver Gummiartikel ist schlecht geworden «, so ist dies in den meisten Fallen gleichbedeutend damit, «das Kautschukstück ist oxydiert". Die Oxydationsfähigkeit des Kautschuks hăngt, wie wir im Nachfolgenden sehen werden, von den verschiedensten Faktoren ab. Die Einwirkung von Sauerstoff auf Kautschuk wurde schon von W. A. Miller and Clark') J. Spiller, Ch, A. Burghard 3), Swan, Dr. C. O. Weber ${ }^{4}$ ), Dr. Henriques, Edgar Herbst ${ }^{\circ}$ und in ganz hervorragender Weise von Prof. Harries ) studiert.

Allgemein bekannt ist es den Gummifabrikanten, daß der gequollene Kautschuk, sogenannte „Kautschuklösung ", viel rascher oxydiert als Stückkautschuk, weshalb man solche Kautschuklösungen in den Fabriken für die Spreadingmaschinen imöglichst rasch verarbeiten muB, weil man sonst keine haltbaren gummierten Stoffe erhält.

In der Technik nennt man den langsamen OxydationsprozeB, welcher durch den Luftsauerstoff bei Gegenwart von Sonnenstrahlen vor sich geht, das »Sonnenbrechen . Es ist oft nötig, die Neigung von Kautschukartikeln zum Sonnenbrechen zu prüfen; zu diesem Zwecke setzt man die Proben längere Zeit dem direkten Sonnenlichte aus und vergleicht sie dann mit den im Dunkeln gehaltenen Stücken von gleichem Material. Diese Methode ist langwierig und

†) Eigenbericht über einen auf der Naturforscherversanumung in Dresden gehaltenen Vortrag.

1) ]. chem. soc. (1865), 18, 280.

a) Dingl. pol. 1. 199, 511 .

4) Gummi-Zeitung Dresden (1903), Nr. 47.

5) Berl. Ber. (1900), Jahrg. 39, Nr. 2, S, 523- 525.

o) Beri. Ber. 37, 2708 (1904); Berl. Ber. 38, 1195 (1905). von den Witterungsverhăltnissen abhängig. Dr. Weber ${ }^{7}$ ) hat deshalb ein bequemeres Laboratoriumsverfahren angegeben, welch es di es elben Prüfungsdienste leistet und darin besteht, daß man gewogene Proben 2 Tage lang in ein 14 Tage altes Gemisch von $20 \mathrm{~g}$ Aceton und $60 \mathrm{ccm} 20$ proz. Wasserstoffsuperoxyd taucht, dann mit Aceton wäscht, bei $100^{\circ} \mathrm{C}$ trocknet und die Cewichtszunahme an Sauerstoff bestimmt, welche ein MaB für die Neigung zum Sonnenbrechen darstellt.

Eine bedeutend einfachere Methode, mit welcher man große Reihen von Kautschukoxydationsprüfungen ausführen kann, wurde von mir ausgearbeitet. Die Oxydation der Proben wird durch zweieinhalbtägiges Behandeln derselben bei $100 \circ \mathrm{C}$ mit Sauerstoff in einem eigens dazu konstruierten Apparat ausgeführt. Die Gewiclitszunahme beim Rohkautschuk nach der Oxydation gibt ein MaB für die Oüte des betreffenden Rohgummis. Hat man ein Kautschukmuster zu untersuchen, danı verfährt man genau so wie mit Rohkautschuk. Verschiedene Rohkautschuksorten oxydieren verschieden schnell. Vom Harzgehalte wird die Oxydationsgeschwindigkeit wenig beeinfluBt. Oxydationsanalysen an im Laboratorium eutharzten und an den gleichen unentharzten Kautschuken angestellt zeigten deutlich, daß entharzte Rohkautschuke stärker oxydieren als unentharzte.

Verschiedene Zusätze zu Kautschukmischungen in verschiedener Menge beigefügt zeigen verschiedene Oxydation des Kautschuks. Zinkoxydzusatz bewirkt erhöhte Oxydationsfähigkeit im Vergleich zu eineun Muster, welches oline Zinkoxyd vulkanisiert wurde. Eine Erhöhung des Zinkoxyds bewirkt kaum eine Erhöhung der Oxydationsfăhigkeit.

7) Gummi-Zeitung Dresden, 18. Jahrg., S. 911. 
Erhöhter Zusatz von Baryumsulfat erhöht die Oxydationsfăhigkeit der Mischuing.

Glasmehl verhält sich ziemlich indifferent.

Bei einem Zusatz von Steinkohlenteerpech in verschiedenen Mengen ansteigend verläuft die Oxydation sehr unregelmäßig, zeigt jedoch im allgemeinen keinen großen Unterschied von der von Steinkohlenteerpech freien gleichen Probe.

Erhöhter Faktiszusatz bewirkt eine Herabsetzung der Oxydationsfähigkeit des Kautschuks. Wir haben somit im Faktis ein Mittel, die Oxydationsfähigkeit minderwertiger Kautschuksorten zu verringern.

Ich habe auch Oxydationsversuche an einem Regenerat angestellt, welches 23 Proz. Kautschuksubstanz, an Kautschukgebundenen Schwefel 1,46 Proz. und 0,02 Proz. freien Schwefel enthielt. Es wurde mit verschiedenen Prozentsätzen von Schwefel 44 Minuten auf 4 Atmosphären vulkanisiert. Die Oxydationsfähigkeit dieses Regenerates nahm bei erhöhtem Schwefelzusatz anfangs zu, bei weiterer Erhöhung ab. Dies dürfte jedenfalls aber mit dem großen Gehalt an freiem Schwefel zusammenhängen. Es kommit nämlich bei den Oxydationen vor, wenn sehr viel freier Schwefel vorhanden ist, daB dieser durch den Sauerstoff als Schwefeldioxyd wegoxydiert wird, daß also die Oxydation negativ ausfällt. Für die Praxis hat ein solches Resultat ebenfalls großien Wert, weil ein Produkt, bei welchem der freie Schwefel in so großer Menge vorhanden ist, daf3 er fortoxydiert wird, absolut unrichtig vulkan'siert ist. Ein vulkanisierter Kautschuk soll nur wenig freien Schwetel enthalten. In normalen Fällen ist darin die Oxydation stets positiv.

Interessant ist das Verhaiten der vulkanisierten, im Laboratorium entharzten Kautschuke bei der. Oxydation und ein Vergleich mit den gleichen vulkanisierten, unentharzten Produkten. Es zeigte sich, daß die vulkanisierten entharzten Kautschuke bloß eine Spur rascher oxydieren als die unentharzten vulkanisierten Kautschuke.

Zum Schlusse wollen wir noch einen Vergleich zwischen verschiedenen Kautschuksorten, die mit 4 Proz. Schwefel bis zur knappen Ausvulkanisation einmal bei 4 Atmosphären und ein zweitesmal bei 15 Atmosphären vulkanisiert waren, anstellen. Es zeigt sich, daß eine Vulkanisation bei höherem Atmosphärendruck zu einem Produkte führt, welches oxydationsfähiger ist, als wenn man bei niedrigerem Druck vulkanisiert.

\section{Ueber die Zulässigkeit von Regeneraten zu Gummi- mischungen, aus welchen hygienische Gummiartikel hergestellt werden sollen.}

Von Dr. Rud. Ditmar, Graz. ")

Veranlassung zu diesem Vortrage waren Anfragen von Gummiwarenfabrikanten an mein Laboratorium, ob man regenerierten Kautschuk zu Oummikompnsitionen für hygietrische Artikel verwenden kann.

Bevor wir uns mit dieser Frage beschäftigen, müssen wir uns darüber klar werden, was eigentlich Regenerater sind. Die Frage läBt sich nicht so einfach beantworten, denn es existieren eine große Anzahl von patentierten Regenerationsverfahren, welche sehr verschieden sind und infolgedessen auch verschiedene Quali-. täten von regeneriertem Gummi liefern. Um die zahlreichen Regenerationsverfahren eingehend z.u behandeln, dazu ist die Zeit zu knapp bemessen. Es soll darüber ausführlich in der Cöthener Chemíkerzeitung referiert werden. An dieser Stelle wollen wir uns bloß mit einer

') Eigenbericht eines auf der Naturforscherversammiung zu Dresdeu gehaltenen Vortrags. kurzen Einteilung begnügen, in welcher die zahlreichen Patente nach gewissen Oesichtspunkten geordnet aufgeführt werden.

I. Säureverfahren:

Clapp,

Brown,

Mitchell.

II. Neutrales Verfahren:

Albert Theilgaard, Kopenhagen, D. R. P. Nr. 112017, 150271 und 112500, K1. 39.

III. Alkali-Verfahren:

Adolf Kittel, Wien, D. R. P. Nr. 172866 , K1. 39 b, Gruppe 2 .

Arthur Marks, Akron (Ohio V. St. v. Amer.),

D. R. P. Nr. 119127 .

A/S Gummi-Regenerations-Geselisch. (System Reesen-Steenstrup), Franz. Patent Nr. 351816 (1905).

H. F. Gregory und T. M. Thorn, Engl. Pat. Nr. 8378. 